\title{
Is Therapeutic Hypothermia Viable in Our Region as a New Option for Sudden Cardiac Arrest Caused by Acute Myocardial Infarction?
}

\author{
Bărcan Andreea*, Rat Nora, Orzan Marius \\ University of Medicine and Pharmacy of Tirgu Mures, Clinic of Cardiology, Tirgu Mures, Gheorghe Marinescu street \\ no. 540139, Romania
}

Received: 14 February 2015 / Accepted: 05 April 2015

\section{-TO THE EDITOR OF JCCM}

Therapeutic hypothermia has become a widely accepted option for management of patients with cardiac arrest occurring in the setting of an Acute Myocardial Infarction [1]. Infarct size is one of the major determinants of future evolution of patients with myocardial infarction and every attempt should be made in order to reduce the amount of infarcted, necrotic myocardium. It has been suggested that induction of hypothermia before performing a percutaneous coronary intervention for urgent revascularisation might play a decisive role in reduction of infarct size [1]. In the same time, applying a mild cooling protocol in patients surviving an out-ofhospital cardiac arrest might significantly improve the rates of neurologically intact survivals on long term [2].

It has been proved that via application of therapeutic hypothermia protocols, a 7\% reduction in cerebral metabolism can be achieved per each $1{ }^{\circ} \mathrm{C}$ of hypothermia, that would further lead to a decrease consumption of glucose and oxygen and prevention of neuronal injury [2]. All these are strongly correlated with the evolution of the neurological status in the post-resuscitation period.

The current guidelines for therapeutic hypothermia established a class IIa indication for applying a mild cooling protocol to $32-34^{\circ} \mathrm{C}$ for $12-24$ hours in unconscious patients surviving an out-of-hospital cardiac arrest [2].
In animal models, it has also been proved that some substances could play a role in pharmacologically-induced hypothermia, which has been proved to be superior to the classical physical hypothermia [3].

The HYPERION trial, a multicenter, randomized, controlled study, will try to find out, on a large number of patients, whether therapeutic hypothermia is clearly associated with superior results as compared with nohypothermia, in unconscious patients resuscitated after a cardiac arrest [4].

Despite the large acceptance of this new therapy in the recent years and despite the clear need for new approaches in these critically ill patients, hypothermia has not been introduced yet as a viable therapeutic strategy in our country. Even though the number of cases with acute myocardial infarction in Romania is one of the highest one in Europe and well-organized networks started to be implemented for treatment of patients with ST-elevation Acute Myocardial Infarction (STEMI networks) in our region, no functional system is implemented yet in these networks that would ensure a fast recovery of the functional status in critical patients with sudden cardiac arrest occurring in the context of such a STEMI event.

One of the main problems recorded in these local STEMI network is the time delay from the onset of symptoms to stent implantation in the culprit coronary artery. A well-known paradigm in interventional

\footnotetext{
* Correspondence to: Bărcan Andreea, University of Medicine and Pharmacy of Tirgu Mures, Clinic of Cardiology, Tirgu Mures, Gheorghe Marinescu street no. 50; e-mail: barcan_a@yahoo.com
} 
cardiology states that "time is muscle" underlining the role of urgent revascularization for recovery of larger amounts of myocardium. For cases with cardiac arrest, this paradigm can be also rephrased as "time is brain", indicating the detrimental role of prolonged hypoxia for the function of brain.

In the context of a well-functioning STEMI network in our region, that provides all the necessary key elements for rapid access to the patient and urgent transportation to the catheterisation laboratory, the number of patients diagnosed with STEMI and arriving in time to the catheterisation laboratory is increasing every month [5]. However, a significant proportion of patients arrive in the hospital intubated, following a successful resuscitation for cardiac arrest with ischaemic cause. In many of these cases, primary PCI is successfully performed and the coronary artery is opened and stented, however the degree of neurological deterioration can have a more significant impact on the future quality of life of these patients.

Therefore we believe that implementation of this treatment option represented by controlled hypothermia should represent one of the first priorities for all the healthcare-related systems (STEMI networks, patient organizations, hospitals, public ambulances, etc), on regional and national level as well. For this, a very important role could have the dissemination of the current knowledge in this field, including publication of scientific articles on this topic in top journals addressing the critically ill patients.

\section{口CONFLICT OF INTEREST}

None

\section{DEFERENCES}

1. Schwartz BG, Kloner RA, Thomas JL, et al - Therapeutic hypothermia for acute myocardial infarction and cardiac arrest. Am J Cardiol. 2012 Aug 1;110(3):461-6.

2. Luscombe Mark, Andrzejowsk John - Clinical applications of induced hypothermia. Contin Educ Anaesth Crit Care Pain, 2006, 6(1):23-27, doi: 10.1093/bjaceaccp/mki0.

3. Katz LM, Frank JE, Glickma1. n LT, Jr GM, Lambdrt BH, Gordon CJ. Effect of a Pharmacologically Induced Decrease in Core Temperature in Rats Resuscitated from Cardiac Arrest. Resuscitation. 2015 Apr 20. pii: S0300-9572(15)00159-8. doi: 10.1016/j.resuscitation.2015.04.009. [Epub ahead of print

4. Lascarrou JB, Meziani F, Le Gouge A, et al - Therapeutic hypothermia after nonshockable cardiac arrest: the HYPERION multicenter, randomized, controlled, assessor-blinded, superiority trial. Scand J Trauma Resusc Emerg Med. 2015 Mar 7;23(1):26. doi: 10.1186/s13049-015-0103-5.

5. Benedek Imre, Gyongyosi Mariann, Benedek Theodora A prospective regional registry of ST-elevation myocardial infarction in Central Romania: Impact of the Stent for Life Initiative recommendations on patient outcomes. American Heart J, 2013, 166;3:457-465. 